Journal of Universal Mathematics

Vol.2 No.1 PP.1-7 (2019)

ISSN-2618-5660

\title{
STATISTICAL CONVERGENCE OF WEIGHT $g$ IN A LOCALLY SOLID RIESZ SPACE
}

\author{
SUKRAN KONCA AND ERGIN GENC
}

\begin{abstract}
In this work, we introduce the notions of statistical convergence and lacunary statistical convergence of weight $g$ in a locally solid Riesz space and establish some inclusion relations.
\end{abstract}

\section{INTRODUCTION}

The Riesz space was first introduced by F. Riesz in 1928, at the International Mathematical Congress in Bologna [1]. Soon after, in the mid-thirties, H. Freudental [2] and L. V. Kantrovich [3] independently set up the axiomatic foundation and derived a number of properties dealing with the lattice structure of Riesz space. Riesz space have many applications in measure theory, operator theory and optimization. They also have some applications in economics [4], we may refer to $[5,6,7,8]$.

Recently, Balcerzak et al. [9] show that one can further extend the concept of natural or asymptotic density (as well as natural density of order $\alpha$ ) by considering natural density of weight $g$ where $g: N \rightarrow[0, \infty]$ is a function with $\lim _{n \rightarrow \infty} g(n)=$ $\infty$ and $\frac{n}{g(n)}$ does not go to 0 as $n \rightarrow \infty$ (Throughout the paper by N, R and C, we will denote the set of all natural, real and complex numbers, respectively). We denote by $G$, the set of all such functions $g$.

In this work, we introduce the notions of statistical convergence and lacunary statistical convergence with respect to weight $g$ in locally solid Riesz space and establish some inclusion relations.

\section{Definitions and Preliminaries}

Let $E \subseteq N$. Then the natural density of $E$ is denoted by $\delta(E)$ and defined by

$$
\delta(E)=\lim _{n \rightarrow \infty}|\{k \in E: k \leq n\}|,
$$

where the vertical bars denote the cardinality of the respective set [10].

Date: Review December 1, 2018, accepted January 14, 2019.

2000 Mathematics Subject Classification. Primary 40G15, Secondary 460D25, 40A35, 46A40, $40 \mathrm{~A} 05$

Key words and phrases. density, locally solid Riesz space, statistical convergence, topological vector space, weight $g$. 
A sequence $x=\left(x_{k}\right)$ of real numbers is said to be statistically convergent to $x_{0}$ if for arbitrary $\epsilon>0$, the set $A(\epsilon)=\left\{n \in N:\left|x_{n}-x_{0}\right| \geq \epsilon\right\}$ has natural density [9].

Let $g: N \rightarrow[0, \infty)$ be a function with $\lim _{n \rightarrow \infty} g(n)=\infty$. The upper density of weight $g$ was defined by the formula

$$
\bar{d}_{g}(A)=\limsup _{n \rightarrow \infty} \frac{A(1, n)}{g(n)}
$$

for $A \subseteq N$ where $A(1, n)$ denotes the cardinality of the set $A \cap[1, n]$. If the $\lim _{n \rightarrow \infty} A(1, n) / g(n)$ exists then we say that the density of weight $g$ of the set $A$ exists and we denote it by $d_{g}(A)$.

Let $X$ be a real vector space and " $\leq$ " be a partial order on this space if it satisfies the following properties:

(1) $\forall x, y \in X$ and $y \leq x$ imply $y+z \leq x+z$ for each $z \in X$,

(2) $\forall x, y \in X$ and $y \leq x$ imply $\alpha y \leq \alpha x$ for each $\alpha \geq 0$.

In addition, if $X$ is lattice with respect to the partial order, then $X$ is said to be a Riesz space (or a vector lattice) [7].

A subset $S$ of a Riesz space $X$ is said to be solid if $y \in S$ and $|x| \leq|y|$ implies $x \in X$.

A topological vector space $(X, \tau)$ is a vector space $X$ which has a linear topology $\tau$ such that the algebraic operations of additions and scalar multiplication in $X$ are continuous.

Every linear topology $\tau$ on a vector space $X$ has a base $\mathcal{N}_{\text {sol }}$ for the neighbourhoods of zero satisfying the following properties:

(1) Each $Y \in \mathcal{N}_{\text {sol }}$ is a balanced set, that is, $\alpha x \in Y$ holds for all $x \in Y$ and every $\alpha \in R$ with $|\alpha| \leq 1$.

(2) Each $Y \in \mathcal{N}_{\text {sol }}$ is an absorbing set, that is, for every $x \in X$ there exists $\alpha>0$ such that $\alpha x \in Y$.

(3) For each $Y \in \mathcal{N}_{\text {sol }}$, there exists some $W \in Y$ with $W+W \subseteq Y$.

A linear topology $\tau$ on a Riesz space $X$ is said to be locally solid Riesz space if $\tau$ has a base at zero consisting of solid sets. A LSRS $(X, \tau)$ is a Riesz space equipped with a locally solid topology $\tau$.

Throughout the paper, the symbol $\mathcal{N}_{\text {sol }}$ will denote any base at zero consisting of solid sets and satisfying the above conditions (1), (2), (3) in a locally solid Riesz topology $\tau$. For abbreviation, here and in where follows, we shall write a word "LSRS" instead of a locally solid Riesz space and we mean $\lim _{k \rightarrow \infty} x_{k}$ by $\lim x$ for brevity.

Let $(X, \tau)$ be a locally solid Riesz space. A sequence $x=\left(x_{k}\right)$ in $X$ is said to be $S(\tau)$-convergent to an element $x_{0}$ in $X$ if for each $\tau$-neighbourhood $V$ of zero

$$
\delta\left(\left\{k \in N: x_{k}-x_{0} \notin V\right\}\right)=0
$$

i.e.,

$$
\lim _{n \rightarrow \infty} \frac{1}{n}\left|\left\{k \leq n: x_{k}-x_{0} \notin V\right\}\right|=0 .
$$

In this case, we write $S(\tau)-\lim x=x_{0}$ or $\left(x_{k} \stackrel{S(\tau)}{\rightarrow} x_{0}\right.$ [8].

By a lacunary sequence, we mean an increasing integer sequence $\theta=\left(k_{r}\right)$ such that $k_{0}=0$ and $h_{r}=k_{r}-k_{r-1} \rightarrow \infty$ as $r \rightarrow \infty$. Throughout the paper, the 
intervals determined by $\theta$ will be denoted by $I_{r}:=\left(k_{r-1}, k_{r}\right]$ and the ratio $k_{r} / k_{r-1}$ will be abbreviated by $q_{r}$.

Let $\theta$ be a lacunary sequence and $(X, \tau)$ be a LSRS. Then a sequence $x=\left(x_{k}\right)$ in $X$ is said to be lacunary statistically $\tau$-convergent to the element $x_{0} \in X$ if for every $\tau$-neighbourhood $V$ of zero, $\delta(K)$, where $K=\left\{k \in N: x_{k}-x_{0} \notin V\right\}$, i.e.,

$$
\lim _{r \rightarrow \infty} \frac{1}{h_{r}}\left|\left\{k \in I_{r}: x_{k}-x_{0} \notin V\right\}\right|=0 .
$$

In this case we write $S_{\theta}(\tau)-\lim x=x_{0}$ or $S_{\theta}(\tau)-\lim x_{k}=x_{0}$ or $x_{k} \stackrel{S_{\theta}(\tau)}{\rightarrow} x_{0}[10]$.

\section{Main Results}

Definition 3.1. Let $(X, \tau)$ be a locally solid Riesz space and $\left(x_{n}\right)$ be a sequence in $X$. We say that $\left(x_{n}\right)$ is statistically $\tau$-convergent of weight $g$ to $x_{0} \in X$ or $S_{g}(\tau)$-convergent to $x_{0}$ provided that for every $\tau$-neighbourhood $U$ of zero,

$$
\lim _{n \rightarrow \infty} \frac{1}{g(n)}\left|\left\{k \leq n: x_{k}-x_{0} \notin U\right\}\right|=0
$$

holds. We denote this by $S_{g}(\tau)-\lim x_{n}=x_{0}$ or $\left(x_{k} \stackrel{S_{g}(\tau)}{\rightarrow} x_{0}\right.$ briefly). The class of all sequences which are statistically $\tau$-convergent of weight $g$ will be denoted by $S_{g}(\tau)$.

Remark 3.2. For $g(n)=n^{\alpha}$ and $X=R$ the definition given above reduces to statistical convergence of order $\alpha$ [11].

Definition 3.3. Let $(X, \tau)$ be a locally solid Riesz space. We say that $x=\left(x_{k}\right)$ in $X$ is said to be $S_{g}(\tau)$-bounded if for every neighbourhood $V$ of zero, there exists some $\alpha>0$ such that,

$$
\lim _{n \rightarrow \infty} \frac{1}{g(n)}\left|\left\{k \leq n: x_{k} \notin V\right\}\right|=0 .
$$

Theorem 3.4. Let $(X, \tau)$ be a Hausdorff LSRS and $x=\left(x_{k}\right), y=\left(y_{k}\right)$ be two sequences in $X$. Then the followings hold:

(1) If $S_{g}(\tau)-\lim x_{k}=x_{0}$ and $S_{g}(\tau)-\lim x_{k}=y_{0}$, then $x_{0}=y_{0}$.

(2) If $S_{g}(\tau)-\lim x_{k}=x_{0}$, then $S_{g}(\tau)-\lim \alpha x_{k}=\alpha x_{0}$, for every $\alpha \in R$,

(3) If $S_{g}(\tau)-\lim x_{k}=x_{0}$ and $S_{g}(\tau)-\lim y_{k}=y_{0}$, then $S_{g}(\tau)-\lim x_{k}+y_{k}=$ $x_{0}+y_{0}$.

Proof. (1) Suppose that $S_{g}(\tau)-\lim x_{k}=x_{0}$ and $S_{g}(\tau)-\lim x_{k}=y_{0}$. Let $V$ be any $\tau$-neighbourhood of zero. Then there exists a $Y \in \mathcal{N}_{\text {sol }}$ such that $Y \subseteq V$. Choose $W \in \mathcal{N}_{\text {sol }}$ such that $W+W \subseteq Y$. Since $S_{g}(\tau)-\lim x_{k}=x_{0}$ and $S_{g}(\tau)-\lim x_{k}=y_{0}$, then we have $d_{g}\left(G_{1}\right)=d_{g}\left(G_{2}\right)=1$ where

$$
\begin{aligned}
& G_{1}=\left|\left\{k \leq n: x_{k}-x_{0} \in W\right\}\right|, \\
& G_{2}=\left|\left\{k \leq n: x_{k}-y_{0} \in W\right\}\right| .
\end{aligned}
$$

Now let $G=G_{1} \cap G_{2}$. Then we have

$$
x_{0}-y_{0}=x_{0}-x_{k}+x_{k}-y_{0} \in W+W \subseteq Y \subseteq V
$$

for every $k \in G$. Hence for every $\tau$-neighbourhood $V$ of zero we have $x_{0}-$ $y_{0} \in V$. Since $(X, \tau)$ is Hausdorff, the intersection of all $\tau$-neighbourhood $V$ of zero is the singleton set $\{\theta\}$. Thus we get $x_{0}-y_{0}=\theta$, i.e., $x_{0}=y_{0}$. 
(2) Let $S_{g}(\tau)-\lim x_{k}=x_{0}$ and let $V$ be an arbitrary $\tau$-neighbourhood of zero. Then there exists $Y \in \mathcal{N}_{\text {sol }}$ such that $Y \subseteq V$. Since $S_{g}(\tau)-\lim x_{k}=x_{0}$, we have

$$
\lim _{n \rightarrow \infty} \frac{1}{g(n)}\left|\left\{k \leq n: x_{k}-x_{0} \in Y\right\}\right|=1 .
$$

Let $|\alpha| \leq 1$. Since $Y$ is balanced, $x_{n}-x_{0} \in Y$ implies that $\alpha\left(x_{n}-x_{0}\right) \in Y$ for every $\alpha \in R$ with $|\alpha| \leq 1$. Hence we have

$$
\begin{aligned}
\left\{k \leq n: x_{k}-x_{0} \in Y\right\} & \subseteq\left\{k \leq n: \alpha\left(x_{n}-x_{0}\right) \in Y\right\} \\
& \subseteq\left\{k \leq n: \alpha\left(x_{k}-x_{0}\right) \in V\right\} .
\end{aligned}
$$

Thus we get

$$
\lim _{n \rightarrow \infty} \frac{1}{g(n)}\left|\left\{k \leq n: \alpha\left(x_{k}-x_{0}\right) \in Y\right\}\right|=1,
$$

for each $\tau$-neighbourhood $V$ of zero. Now let $|\alpha|>1$ and $\llbracket \alpha \rrbracket$ be the smallest integer greater than or equal to $\alpha$. There exists a $W \in \mathcal{N}_{\text {sol }}$ such that $\llbracket \alpha \rrbracket W \subseteq Y$. Since $S_{g}(\tau)-\lim x_{n}=x_{0}$, we have $d(K)=1$, where

$$
K=\left|\left\{k \leq n: x_{k}-x_{0} \in W\right\}\right| .
$$

Then we have

$$
\left|\alpha x_{n}-\alpha y_{0}\right|=|\alpha|\left|x_{n}-x_{0}\right| \leq \llbracket \alpha \rrbracket\left|x_{n}-x_{0}\right| \in W \subseteq Y \subseteq V
$$

for each $n \in K$. Since the set $V$ is solid, we have $\alpha x_{n}-\alpha x_{0} \in Y$ and so $\alpha x_{n}-\alpha x_{0} \in V$ for each $n \in K$. Thus we get

$$
\lim _{n \rightarrow \infty} \frac{1}{g(n)}\left|\left\{k \leq n: x_{k}-x_{0} \in V\right\}\right|=1
$$

for each $\tau$-neighbourhood $V$ of zero. Hence $S_{g}(\tau)-\lim \alpha x_{k}=\alpha x_{0}$ for every $\alpha \in R$.

(3) Let $V$ be an arbitrary $\tau$-neighbourhood of zero. Then there exists $Y \in \mathcal{N}_{\text {sol }}$ such that $Y \subseteq V$. We choose $W \in \mathcal{N}_{\text {sol }}$ such that $W+W \subseteq Y$. Since $S_{g}(\tau)-\lim x_{n}=\alpha x_{0}$ and $S_{(\bar{N}, \theta)}^{I}(\tau)-\lim y=y_{0}$, we have $d\left(B_{1}\right)=d\left(B_{2}\right)=$ 1 where

$$
B_{1}=\left\{k \leq n: x_{k}-x_{0} \in W\right\}
$$

and

$$
B_{2}=\left\{k \leq n: x_{k}-x_{0} \in W\right\} .
$$

Now let $B=B_{1} \cap B_{2}$. Hence we have $d(B)=1$ and

$$
\left(x_{n}+y_{n}\right)-\left(x_{0}-y_{0}\right)=\left(x_{n}-x_{0}\right)+\left(y_{n}-y_{0}\right) \in W+W \subseteq Y \subseteq V
$$

for each $n \in B$. Thus we get

$$
\lim _{n \rightarrow \infty} \frac{1}{g(n)}\left|\left\{k \leq n:\left(x_{n}-x_{0}\right)+\left(y_{n}-y_{0}\right) \in V\right\}\right|=1 .
$$

Since $V$ is arbitrary, we have $S_{g}(\tau)-\lim x_{k}+y_{k}=x_{0}+y_{0}$.

Theorem 3.5. Let $(X, \tau)$ be a locally solid Riesz space. If a sequence $x=\left(x_{k}\right)$ is $S_{g}(\tau)$-convergent, then $x=\left(x_{k}\right)$ is $S_{g}(\tau)$-bounded. 
Proof. Let $S_{g}(\tau)-\lim x_{k}=x_{0}$. Let $V$ be an arbitrary $\tau$-neighbourhood of zero. Then there exists a $Y \in \mathcal{N}_{\text {sol }}$ such that $Y \subseteq V$. Let us choose $W \in \mathcal{N}_{\text {sol }}$ such that $W+W \subseteq Y$. Since $x_{k} \stackrel{S_{g}(\tau)}{\rightarrow} x_{0}$, we have $d(K)=0$, where

$$
K=\left|\left\{k \leq n: x_{k}-x_{0} \in V\right\}\right| .
$$

Since $W$ is absorbing, there exists a $a>0$ such that $a x_{0} \in W$. Let $b$ be such that $b \leq 1$ and $b \leq a$. Since $W$ is solid and $\left|b x_{0}\right| \leq\left|a x_{0}\right|$, we have $b x_{0} \in W$. Since $W$ is balanced, $x_{k}-x_{0} \in W$ implies that $b\left(x_{k}-x_{0}\right) \in W$. Then we have

$$
b x_{n}=b\left(x_{n}-x_{0}\right)+b x_{0} \in W+W \subseteq Y \subseteq V
$$

for each $n \in N \backslash K$ and thus we get

$$
\lim _{n \rightarrow \infty} \frac{1}{g(n)}\left|\left\{k \leq n: b x_{k} \notin V\right\}\right|=0 .
$$

Consequently, $\left(x_{n}\right)$ is $S_{g}(\tau)$-bounded.

Theorem 3.6. Let $(X, \tau)$ be a locally solid Riesz space. If $\left(x_{k}\right),\left(y_{k}\right),\left(z_{k}\right)$ are sequences such that;

(1) $x_{k} \leq y_{k} \leq z_{k}$, for all $k \in N$,

(2) $S_{g}(\tau)-\lim x_{k}=x_{0}=S_{g}(\tau)-\lim z_{k}$, then $S_{g}(\tau)-\lim x_{k}=x_{0}$.

Proof. Let $V$ be an arbitrary $\tau$-neighbourhood of zero. Then there exists $Y \in \mathcal{N}_{\text {sol }}$ such that $Y \subseteq V$. Choose $W \in \mathcal{N}_{\text {sol }}$ such that $W+W \subseteq Y$. From the condition (2), we have $S_{g}(\tau)\left(E_{1}\right)=1=S_{g}(\tau)\left(E_{2}\right)$ where

$$
E_{1}=\left\{k \leq n: x_{k}-x_{0} \in W\right\}
$$

and

$$
E_{2}=\left\{k \leq n: z_{k}-x_{0} \in W\right\} .
$$

Also, we get $S_{g}(\tau)\left(E_{1} \cap E_{2}\right)=1$ and from (1) we have

$$
x_{k}-x_{0} \leq y_{k}-x_{0} \leq z_{k}-x_{0}
$$

for all $k \in N$. This implies that for all $k \in E_{1} \cap E_{2}$, we get

$$
\left|y_{k}-x_{0}\right| \leq\left|z_{k}-x_{0}\right|+\left|x_{k}-x_{0}\right| \in W+W \subseteq Y \subseteq V .
$$

Since $Y$ is solid, we have $y_{k}-y_{0} \in V$. Thus

$$
\lim _{n \rightarrow \infty} \frac{1}{g(n)}\left|\left\{k \leq n: y_{k}-x_{0} \in V\right\}\right|=1
$$

for each $\tau$-neighbourhood $V$ of zero. Hence $S_{g}(\tau)-\lim x_{k}=x_{0}$.

\section{Lacunary Statistical Convergence of Weight $g$ in a Locally Solid RIESZ SPACE}

In this section, we define lacunary statistical convergence of weight $g$ in a locally solid Riesz space and we examine some inclusion relations.

Definition 4.1. Let $\theta$ be a lacunary sequence. A sequence $x=\left(x_{k}\right)$ is said to be lacunary statistically convergent of weight $g$ to $x_{0}$ or $S_{g}^{\theta}(\tau)$-convergent to $x_{0}$ if for every $\tau$-neighbourhood $U$ of zero,

$$
\lim _{n \rightarrow \infty} \frac{1}{g\left(h_{r}\right)}\left|\left\{k \in I_{r}: x_{k}-x_{0} \notin U\right\}\right|=0
$$


holds. We denote this by $S_{g}^{\theta}(\tau)$ - $\lim x_{n}=x_{0}$ (or $x_{k} \stackrel{S_{g}^{\theta}(\tau)}{\rightarrow} x_{0}$ briefly). The class of all sequences which are lacunary statistically $\tau$-convergent of weight $g$ will be denoted by $S_{g}^{\theta}(\tau)$.

Definition 4.2. Let $(X, \tau)$ be a locally solid Riesz space and $\theta$ be a lacunary sequence. We say that $x=\left(x_{k}\right)$ in $X$ is said to be $S_{g}^{\theta}(\tau)$-bounded if for every neighbourhood $V$ of zero, there exists some $\alpha>0$ such that,

$$
\lim _{r \rightarrow \infty} \frac{1}{g\left(h_{r}\right)}\left|\left\{k \leq I_{r}: x_{k} \notin V\right\}\right|=0
$$

We leave the proofs of the following two theorems to the reader. They can be done in a similar manner as the proofs of Theorem 3.4 and Theorem 3.5.

Theorem 4.3. Let $(X, \tau)$ be a Hausdorff locally solid Riesz space and $\theta$ be a lacunary sequence and $x=\left(x_{k}\right), y=\left(y_{k}\right)$ be two sequences in $X$. Then the followings hold:

(1) If $S_{g}^{\theta}(\tau)-\lim x_{k}=x_{0}$ and $S_{g}^{\theta}(\tau)-\lim x_{k}=y_{0}$, then $x_{0}=y_{0}$.

(2) If $S_{g}^{\theta}(\tau)-\lim x_{k}=x_{0}$, then $S_{g}^{\theta}(\tau)-\lim \alpha x_{k}=\alpha x_{0}$, for every $\alpha \in R$,

(3) If $S_{g}^{\theta}(\tau)-\lim x_{k}=x_{0}$ and $S_{g}^{\theta}(\tau)-\lim y_{k}=y_{0}$, then $S_{g}^{\theta}(\tau)-\lim x_{k}+y_{k}=$ $x_{0}+y_{0}$.

Theorem 4.4. Let $(X, \tau)$ be a locally solid Riesz space and $\theta$ be a lacunary sequence. If a sequence $x=\left(x_{k}\right)$ is $S_{g}^{\theta}(\tau)$-convergent, then $x=\left(x_{k}\right)$ is $S_{g}^{\theta}(\tau)$ bounded.

Theorem 4.5. Let $(X, \tau)$ be a locally solid Riesz space and $\theta$ be a lacunary sequence. If $\liminf \frac{g\left(h_{r}\right)}{g\left(k_{r}\right)}>1$, then $S_{g}(\tau) \subset S_{g}^{\theta}(\tau)$

Proof. Since $\liminf \sin _{r} \frac{g\left(h_{r}\right)}{g\left(k_{r}\right)}>1$, so we can find a $\delta>0$ such that $\frac{g\left(h_{r}\right)}{g\left(k_{r}\right)} \geq 1+\delta$ for sufficiently large values of $r$. Assume that $x_{k} \stackrel{S_{g}(\tau)}{\rightarrow} x_{0}$, hence for every $U$ neighbourhood of zero and for sufficiently large values of $r$ we have

$$
\begin{aligned}
\frac{1}{g\left(k_{r}\right)}\left|\left\{k \leq k_{r}: x_{k}-x_{0} \notin U\right\}\right| \geq \frac{1}{g\left(k_{r}\right)}\left|\left\{k \in I_{r}: x_{k}-x_{0} \notin U\right\}\right| \\
=\frac{g\left(h_{r}\right)}{g\left(k_{r}\right)} \frac{1}{g\left(h_{r}\right)}\left|\left\{k \in I_{r}: x_{k}-x_{0} \notin U\right\}\right| \\
\geq(1+\delta) \frac{1}{g\left(h_{r}\right)}\left|\left\{k \in I_{r}: x_{k}-x_{0} \notin U\right\}\right| .
\end{aligned}
$$

Hence we have $x_{k} \stackrel{S_{g}^{\theta}(\tau)}{\rightarrow} x_{0}$ while taking limit as $n \rightarrow \infty$.

\section{Conclusion}

In this work, we introduce the notions of statistical convergence and lacunary statistical convergence with respect to weight $g$ in a locally solid Riesz space and establish some inclusion relations. One can see that if $\lim \sup _{r} \frac{g\left(h_{r}\right)}{g\left(k_{r}\right)}<\infty$ the inverse of the Theorem 4.5 holds. 


\section{REFERENCES}

[1] F. Riesz, Sur la decomposition des operations fonctionnelles lineaires, In Atti del Congresso Internazionale dei Matematici: Bologna del 3 al 10 de settembre di 1928, pp. 143-148 (1929).

[2] H. Freudenthal, Teilweise geordnete Moduln, K. Akademie van Wetenschappen, Afdeeling Natuurkunde, Proceedings of the Section of Sciences, 39, 647-657 (1936).

[3] L. V. Kantorovich, Concerning the general theory of operations in partially ordered spaces, Dok. Akad. Nauk. SSSR 1, 271-274 (1936).

[4] C. D. Aliprantis, O. Burkinshaw, Locally solid Riesz spaces with applications to economics, American Mathematical Society, Second Edition, USA, 105, (2003).

[5] L. V. Kantorovich, Lineare halbgeordnete Raume, Recueil Mathmatique, 2(44), 121-168 (1937).

[6] W. A. J. Luxemburg and A. C. Zaanen, Riesz spaces, American Elsevier Publishing Company, New York, (1971).

[7] A. C. Zannen, Introduction to Operator Theory in Riesz Spaces, Springer-Verlag, (1997).

[8] H. Albayrak and S. Pehlivan, Statistical convergence and statistical continuity on locally solid Riesz spaces, Topology and its Applications, 159(7), 1887-1893 (2012).

[9] M. Balcerzak, P. Das, M. Filipczak and J. Swaczyna Generalized kinds of density and the associated ideals, Acta Mathematica Hungarica, 147(1), 97-115 (2015).

[10] B. Hazarika, S. A. Mohiuddine and M. Mursaleen. Some inclusion results for lacunary statistical convergence in locally solid Riesz spaces, Iranian Journal of Science and Technology, 38(A1), 61-68 (2014).

[11] R. Çolak and Ç A. Bektaş. $\lambda$-statistical convergence of order $\alpha$, Acta Mathematica Scientia 31(3), 953-959 (2011).

(Sukran Konca) Department of Mathematics, Bitlis Eren University, 13000, Turkey

E-mail address, Sukran Konca: skonca@beu.edu.tr

(Ergin Genc) Bitlis Eren University, Mathematics Institute of Science, 13000, Bitlis, TURKEY

E-mail address, Ergin Genc: ergingenc4444@gmail.com 